\title{
Research Article \\ Effect of Different Polishing Methods on Anodic Titanium Dioxide Formation
}

\author{
Magdalena Jarosz, Joanna Kapusta-Kołodziej, Marian Jaskuła, and Grzegorz D. Sulka \\ Department of Physical Chemistry and Electrochemistry, Faculty of Chemistry, Jagiellonian University in Krakow, \\ Ingardena 3, 30060 Krakow, Poland \\ Correspondence should be addressed to Grzegorz D. Sulka; sulka@chemia.uj.edu.pl
}

Received 10 December 2014; Revised 26 January 2015; Accepted 20 February 2015

Academic Editor: Alireza Khataee

Copyright (C) 2015 Magdalena Jarosz et al. This is an open access article distributed under the Creative Commons Attribution License, which permits unrestricted use, distribution, and reproduction in any medium, provided the original work is properly cited.

\begin{abstract}
Among various methods of synthesis of nanostructured $\mathrm{TiO}_{2}$, a self-organized anodization is the most commonly used and discussed in the literature. However, different methods of pretreatment of Ti before anodic titanium dioxide (ATO) formation are not often addressed. Therefore, various polishing procedures based on mechanical, chemical, electrochemical, and combined electrochemical with chemical pretreatments were examined to establish whether they represent effective methods for smooth $\mathrm{Ti}$ surface preparation before anodization. The ATO layers were prepared via two-step anodization carried out in an ethylene glycol solution containing fluoride ions at $20^{\circ} \mathrm{C}$ and under the anodizing potential of $60 \mathrm{~V}$. The influence of applied polishing method on the cell size, pore diameter, pore circularity, pore density, and porosity of the top ATO layer was studied. In addition, the effect of polishing procedure on cell arrangement in ATO films was also investigated. The quantitative analyses of the regularity of cell arrangement, based on the regularity ratio derived from bottom-view SEM images, showed that the type of polishing procedure does not affect the cell order.
\end{abstract}

\section{Introduction}

Nowadays, scientists are overwhelmingly interested in the fundamental aspects and applications of semiconducting wide bandgap oxide materials. Among various semiconductors, titanium dioxide $\left(\mathrm{TiO}_{2}\right)$ plays an important role because of its chemical stability, nontoxicity, and biocompatibility. It is also an inexpensive material with a very high dielectric constant and interesting photocatalytic activities [1]. Taking the advantages of its properties, titania has recently gained significant attention as a functional material with wide spectrum of photocatalytic $[1,2]$, solar cell $[3,4]$, water splitting [5], antibacterial [6], and sensing applications [7, 8]. It is also recognized as an important biocompatible material for synthetic bone graft substitutes $[9,10]$. Due to the large surface area to volume ratio and the quantum confinement effect, $\mathrm{TiO}_{2}$ nanotubes and nanopore arrays are most frequently fabricated nanostructures [11, 12]. Several different methods, such as sol-gel [13], template-assisted [14], hydrothermal [15], or seeded growth [16], have been used for the fabrication of titania nanostructures. However, highly ordered nanotubular/nanoporous structures with controllable dimensions can only be produced via a self-organized anodization of titanium.

Anodization is a simple electrochemical process used in order to produce thick, nanoporous/nanotubular metal oxide layers on valve metals. The anodic oxidation of metals, especially $\mathrm{Al}$ and $\mathrm{Ti}$, has been studied extensively for various purposes [17-20]. In general, the majority of principles that apply to the preparation of anodic titanium dioxide also apply to the formation of anodic aluminum oxide (AAO). For instance, by changing anodization conditions such as applied voltage [21, 22], water content in the electrolyte [23], temperature and potential sweep rate [24], nanostructures with different pore/tube diameters, and different regularities of the nonstructural architecture can be obtained. Another similarity to $\mathrm{Al}$ anodization is a direct dependence between the length of nanochannels/nanotubes and the anodization time [22]. 
In addition, the arrangement of nanopores/nanotubes can be significantly improved by using a two-step anodization method [25, 26]. However, insufficient research has been made to examine the anodization of $\mathrm{Ti}$.

The main unknown factor in nanostructured titania formation is a surface preparation procedure used before anodizing. The different pretreatment procedures affect the nanopore/nanotube arrangement in as-obtained $\mathrm{TiO}_{2}$. It has been established that anodic $\mathrm{Al}_{2} \mathrm{O}_{3}$ has better arranged nanopores, when the $\mathrm{Al}$ surface is pretreated by annealing or electropolishing the foil used for anodization [27-32]. The most common pretreatment procedures are a mechanical polishing with emery paper, alumina or diamond slurries, and electropolishing in various electrolytes. The electropolishing processes, carried out at the galvanostatic or potentiostatic modes, involve typically the following electrolytes based on (i) sulfuric acid in water or organic solvents with or without the addition of hydrofluoric acid [33-35], (ii) perchloric acid in methanol $[36,37]$, and (iii) perchloric acid and glacial acetic acid [38, 39]. Although the electropolishing process carried out in those electrolytes results in a microscopically bright, clean, and smooth Ti surface, electrolytes used in this process are either toxic or flammable. Therefore, some researchers are focusing on finding less harmful mixtures [40-42].

Certainly, properties of nanostructured anodic $\mathrm{TiO}_{2}$ depend on operating conditions of anodization and the method of preparation of the substrate prior to anodizing. Nevertheless, it is still unclear how the pretreatment of $\mathrm{Ti}$ before anodization improves the arrangement of nanotubes/nanopores. Recently, a few studies regarding the effect of polishing of $\mathrm{Ti}$ and $\mathrm{Ti}$ alloys on the growth of ATO layers have been conducted applying different polishing strategies including mechanical polishing [43-45], chemical polishing [45], and electropolishing [42, 45]. For example, Kim et al. established that highly aligned $\mathrm{TiO}_{2}$ nanotube array can be obtained on a mechanically polished $\mathrm{Ti}$ surface, as the chemical etching of the top nanotube layer is considerable suppressed [44]. The reduced rate of chemical etching of nanotubes is a direct result of the formation of thin rutile layer in the early stage of anodization. In addition, it was found that electropolishing is indispensable for the formation of uniform nanotubular $\mathrm{TiO}_{2}$ structures [42]. On the other hand, the effect of different surface conditions, as a consequence of used different surface polishing procedures, on the nanotube morphology was studied by Lu et al. [45]. Nevertheless, no systematic studies were performed to investigate the influence of applied polishing methods on the $\mathrm{TiO}_{2}$ nanotube arrangement formed by two-step anodization.

Thus, in this work, a simple but relevant comparison of some commonly used procedures for titanium surface preparation before anodization is described. Furthermore, the effect of different polishing methods on the formation of anodic titanium dioxide via two-step anodization was studied. Four different polishing methods were applied: mechanical polishing (MP), chemical polishing (CP), electropolishing (EP), and combined electrochemical and chemical polishing $(\mathrm{EP}+\mathrm{CP})$. The analysis of cell arrangement in ATO layers was investigated for all used procedures of Ti pretreatment.

\section{Experimental}

A titanium foil (99.5\% in purity and $0.25 \mathrm{~mm}$ thick, Alfa Aesar) was precut in coupons $(0.5 \mathrm{~cm} \times 2.0 \mathrm{~cm})$. The samples were degreased in acetone (Chempur) and ethanol (Chempur). Afterwards, four different polishing methods were applied.

The mechanical polishing to a mirror finished was carried out using increasing grits of abrasive paper (70, 120, 600, 800 , and 2000) followed by four different polishing alumina powders of decreasing particle size (mesh: 500, 600, 800, and 1200). Finally the samples were rinsed with water. The chemical polishing was performed by dipping of Ti samples into a stirred mixture of hydrofluoric acid (40 wt.\%, Idalia) and nitric acid (65 wt.\%, Avantor) (1:3 in volume) for $10 \mathrm{s.}$ Then, Ti coupons were rinsed with water and ethanol and dried in the air. The electrochemical polishing was conducted in a mixture (60:15:25 in volume) containing acetic acid (98 wt.\%, Avantor), sulfuric acid (98wt.\%, Avantor), and hydrofluoric acid (40 wt.\%, Idalia) at the constant current density of $140 \mathrm{~mA} \mathrm{~cm}^{-2}$ and $20^{\circ} \mathrm{C}$ for $1 \mathrm{~min}$. Afterwards, the samples were rinsed with water, ethanol, and dried in the air. A combined pretreatment method was based on the electrochemical polishing followed by chemical polishing.

Anodic titanium dioxide layers on $\mathrm{Ti}$ samples were prepared via a two-step anodization in an ethylene glycol solution containing $\mathrm{NH}_{4} \mathrm{~F}$ (0.38 wt.\%, Sigma-Aldrich) and $\mathrm{H}_{2} \mathrm{O}(1.79$ wt. $\%)$ at $20^{\circ} \mathrm{C}$. The process was carried out at $60 \mathrm{~V}$ in a two-electrode cell, with polished $\mathrm{Ti}$ samples as anodes and a $\mathrm{Ti}$ plate as a cathode. The duration of both anodizing steps was $30 \mathrm{~min}$. After the first step of anodization, an adhesive tape was used to remove the oxide layer. All used chemicals were of analytical grade.

The structural and morphological characterizations were performed using a field emission scanning electron microscope (FE-SEM, Hitachi S-4700). The structural features and pore order degree of anodized samples were estimated directly from SEM images by using the scanning probe image processor WSxM 4.0 Develop 12.0 [46] and Image J $1.37 \mathrm{~V}$ software [47].

\section{Results and Discussion}

3.1. Morphology of Ti Surface. Figure 1 shows SEM images of as-received $\mathrm{Ti}$ foil and $\mathrm{Ti}$ samples after different polishing treatments. The as-received $\mathrm{Ti}$ foil has a rough surface with cracks, small pores distributed randomly over the surface, and grains stretched in one direction by the rolling process (Figure 1(a)).

After the MP, the number of pores and cracks is significantly reduced; however, the surface is still rough and the grains on the surface are starched along the rolling direction (Figure 1(b)). The CP in hydrofluoric acid and nitric acid improves considerably the smoothness of the surface (Figure 1(c)). The Ti surface is free from cracks and rough tracks but possesses small domains with nanometer sized grains. Usually, Ti grains have different crystallographic orientations; therefore, the surface is not smooth enough. As reported by $\mathrm{Lu}$ et al., the average roughness and height of 


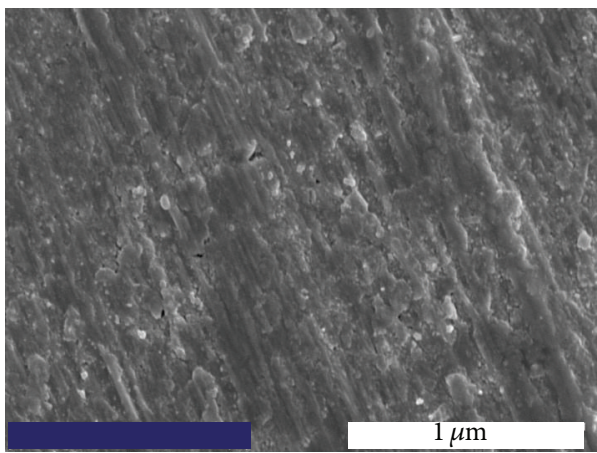

(a) As-received

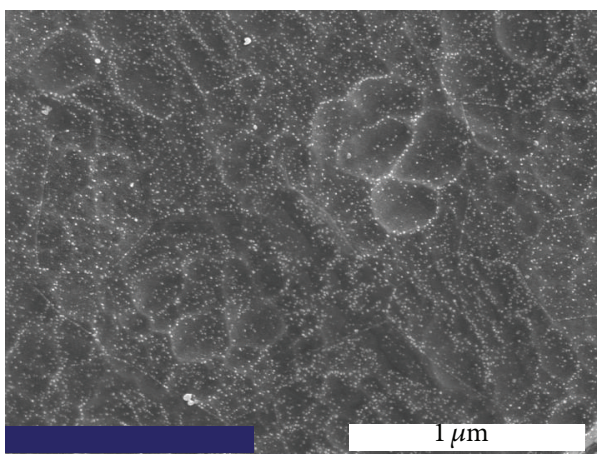

(c) $\mathrm{CP}$

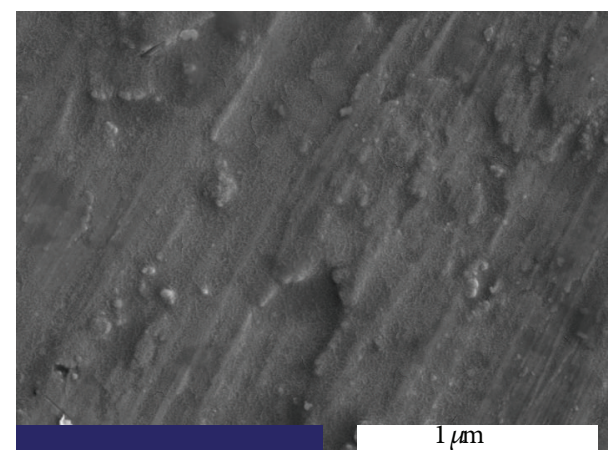

(b) MP

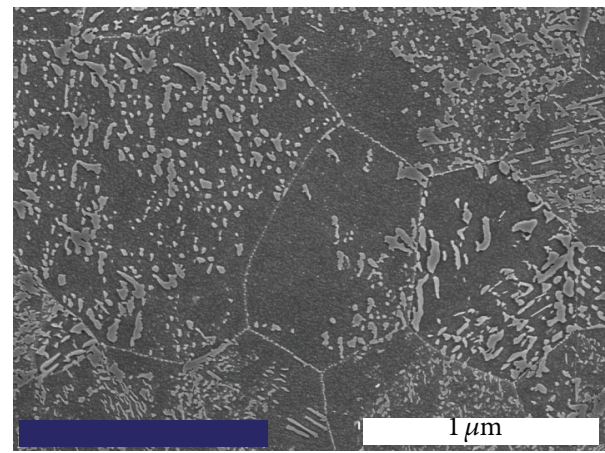

(d) EP

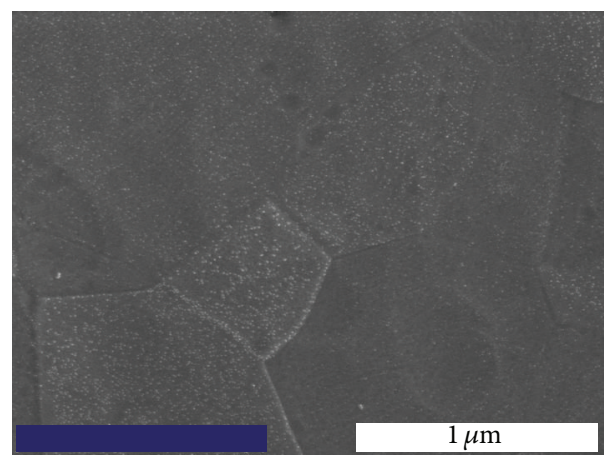

(e) $\mathrm{EP}+\mathrm{CP}$

FIGURE 1: SEM images of Ti (a) as-received and after applied pretreatment procedures, (b) mechanically polished (MP), (c) chemically polished (CP), (d) electrochemically polished (EP), and (e) electrochemically and chemically polished (EP + CP).

the chemically polished surface increase after chemical polishing due to the large periodic undulations on the Ti surface [45]. Moreover, the problem with the chemical polishing might arise due to an uneven stirring of the acidic mixture that, in consequence, causes an unequal contact of the solution with Ti surface. The EP leads to a significantly less rough surface with clearly visible grain boundaries (Figure 1(d)). Although small nanoparticle-like residues are observed, the $\mathrm{Ti}$ surface is much more flat and smooth compared with that after MP. The best result, however, was achieved when the electrochemical and chemical polishing methods were combined together (Figure 1(e)). The surface is flat with smooth grain boundaries and reduced number of residues. Generally, polishing of Ti considerably modifies the surface roughness. From the presented data it is evident that the combined method based on electropolishing followed by chemical polishing gives the best results.

3.2. Polishing Effect on Anodization. Figure 2 shows the comparison of SEM images of the topside (Figures 2(a)2(d)) and backside (Figures 2(e)-2(h)) of $\mathrm{TiO}_{2}$ samples after the second anodizing step, with respect to the four polishing conditions described above. Two-dimensional fast Fourier transforms (2D FFTs) of SEM images were collected for all polishing methods in Figure 1 as well. As expected, different polishing methods affect the quality of received anodic titanium dioxide.

The ATO layer obtained on the MP Ti foil (Figure 2(a)) shows a poorly ordered nanopore array with very rough pore walls. Moreover, the pore diameter varies throughout 


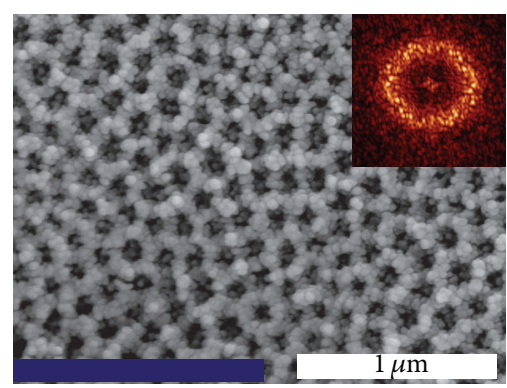

(a) MP

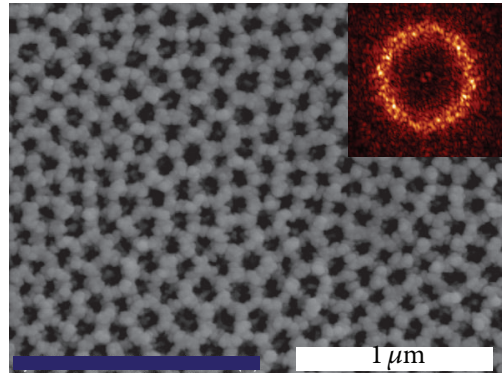

(b) $\mathrm{CP}$

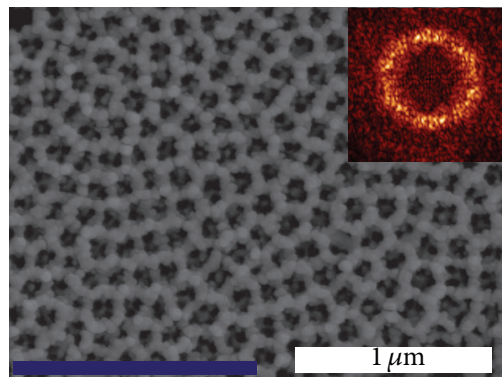

(c) EP

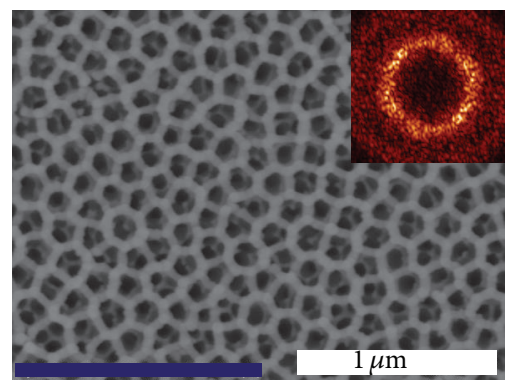

(d) $\mathrm{EP}+\mathrm{CP}$

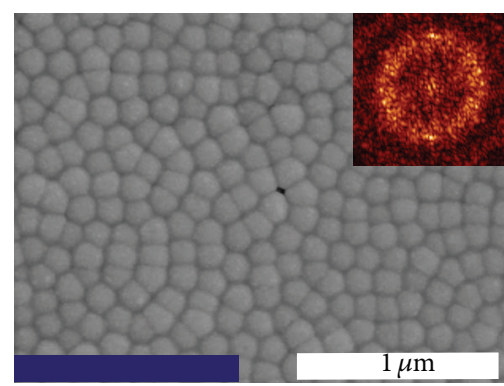

(e) MP

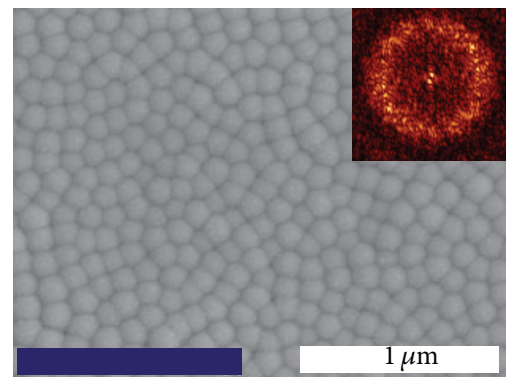

(f) $\mathrm{CP}$

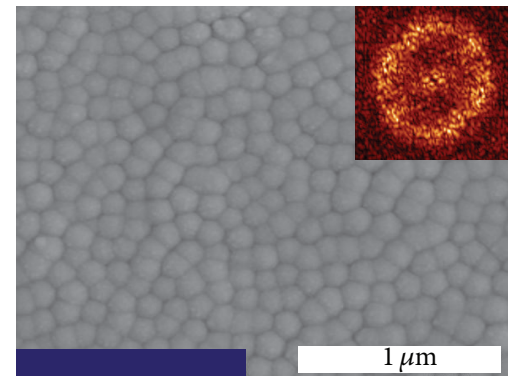

(g) EP

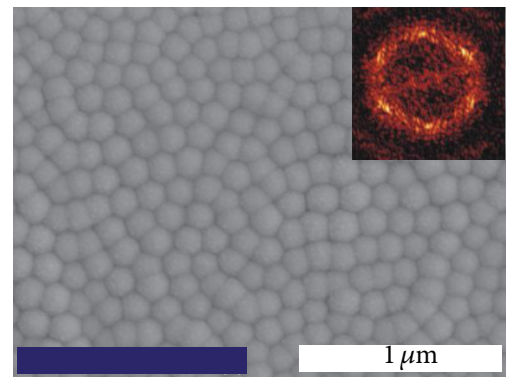

(h) $\mathrm{EP}+\mathrm{CP}$

FIGURE 2: SEM images with FFT patterns for top-side ((a)-(d)) and back-side ((e)-(h)) of $\mathrm{TiO}_{2}$ formed on the Ti foil subjected to different polishing procedures: MP ((a) and (e)), CP ((b) and (f)), EP ((c) and (g)), and EP + CP ((d) and (h)). The two-step anodization was used for ATO formation.

the whole ATO surface and some surface undulations are visible. For chemically polished $\mathrm{Ti}$, the ATO layer shows still a superficial roughness, but the pore structure is slightly improved (Figure 2(b)). The pore size as well as the interpore distance is not uniform and undulations are present on the surface (Figure 2(b)). A very similar ATO structure with similar pore sizes was obtained on the electropolished $\mathrm{Ti}$ foil (Figure 2(c)). The ATO layer grown on Ti foil prepared by the EP + CP shows the uniform structure (Figure 2(d)) with smooth pore walls and undulations are not present. However, the subpores were clearly visible in the pore cells for both the EP and EP + CP methods (Figures 2(c) and 2(d)). Nonetheless, it might be quite an interesting feature when it comes to, for example, catalytic applications (larger 
(a)

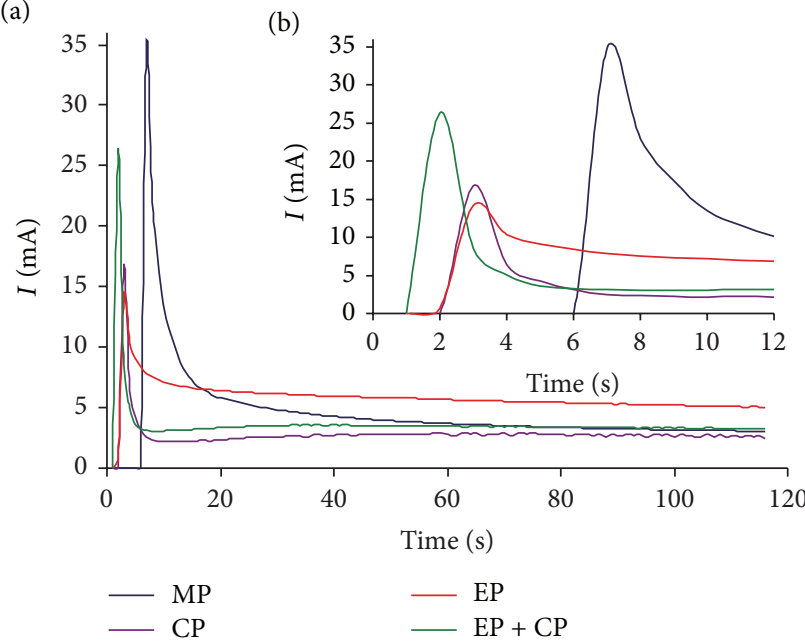

(c)

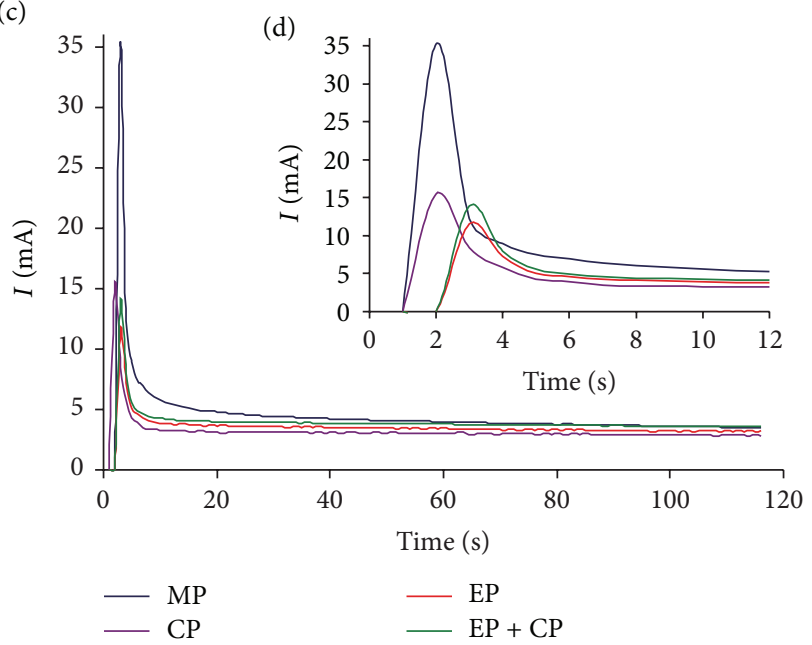

FIGURE 3: Current density versus anodizing time for the first ((a) and (b)) and second ((c) and (d)) anodizing steps. The insets ((b) and (d)) show enlarged curves. The curves were recorded for Ti surfaces pretreated by MP, CP, EP, and EP + CP.

surface area) or cell culturing on ATO surfaces [44, 48]. From the presented FFT images, a qualitative analysis of pore order on the ATO surface is rather difficult to be performed. Almost independently of the polishing method used for $\mathrm{Ti}$ pretreatment, the observed FFT pattern exhibited relatively broad ring-like shapes that indicate large number of domains with nonsharp boundaries between them.

Looking at the backside of ATO layers (Figures 2(e)2(h)), surprisingly, the ATO structures are very similar to each other, independently of the used polishing method. The arrangement of cells is similar and the only visible difference is their size. The biggest size of cells is observed for the sample subjected to the MP pretreatment. The analysis of the FFT patterns provides the similar conclusions regarding the cell order degree. The FFT patterns are blurred and the discshape forms are visible in all cases, independently of the used polishing method. It suggests that the multistep anodizing procedure plays a predominant role in pore ordering. Therefore, the similar cell order can be ascribed to the well-known influence of the second anodizing step on the pore order [e.g., [9]].

In order to better understand how the polishing method of $\mathrm{Ti}$ surface affects the formation of anodic $\mathrm{TiO}_{2}$, the analysis of current-time curves recorded during anodizations was performed. Figure 3 shows the current density versus anodization time curves recorded for the first and second anodizing steps.

The observed evolutions of current density with time are typical for anodization of Ti that leads to the formation of nanoporous $\mathrm{TiO}_{2}[9,48]$. At the beginning of anodization (Figure 3(a)), a compact passive layer was formed on the $\mathrm{Ti}$ surface and the recorded current density was close to zero. After a few seconds (usually 1-2 s), the compactness of passive oxide layer is lost due to the formation of pores and the current density increases. A rearrangement of formed pores on the surface is indicated on the current-time curve by a local maximum that appears typically in $2-3 \mathrm{~s}$. The pore

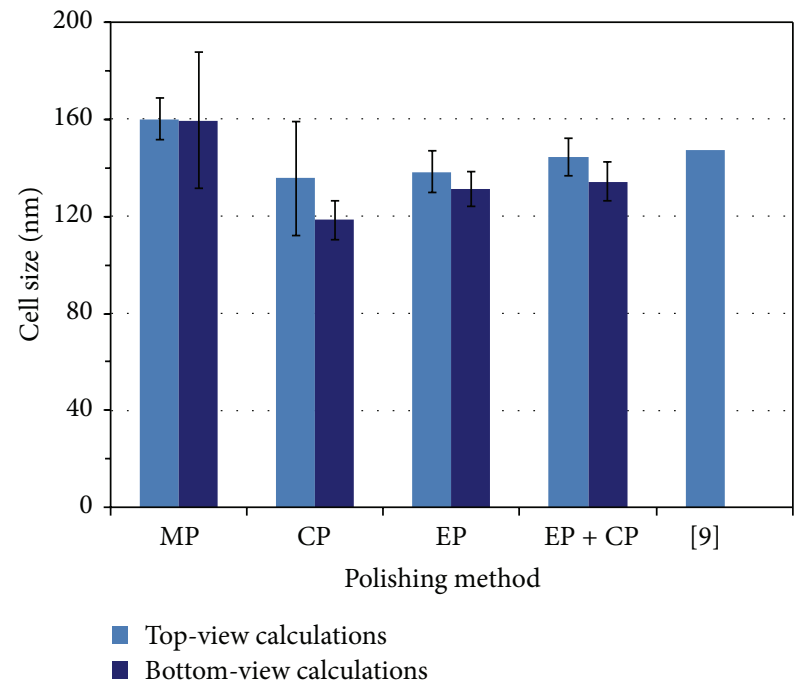

FIGURE 4: Influence of the polishing method on the cell size of anodic titanium dioxide formed by two-step anodization.

rearrangement process results in the formation of network of close-packed pores. After reaching the maximum value, the current density decreases with time until a steady-state value of porous oxide grow is achieved.

For the first anodizing step, the longest time needed to reach the current maximum is observed for MP (Figure 3(b)). It indicates that the pore formation on the MP surface is significantly delayed. On the other hand, the sample pretreated by $\mathrm{EP}+\mathrm{CP}$ reaches the maximum in a very short time. During the second anodizing step, all samples reach the current density maximum at a similar time (2-3 s). It suggests that, independently of the used polishing method, there is no significant difference in the surface state before the second anodization. Importantly, after removal of the oxide layer grown during the first anodizing step, the regular 


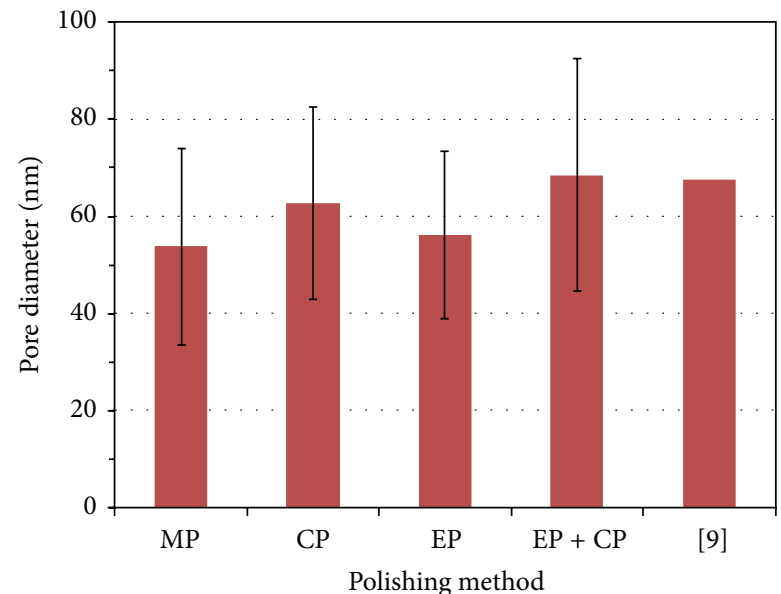

(a)

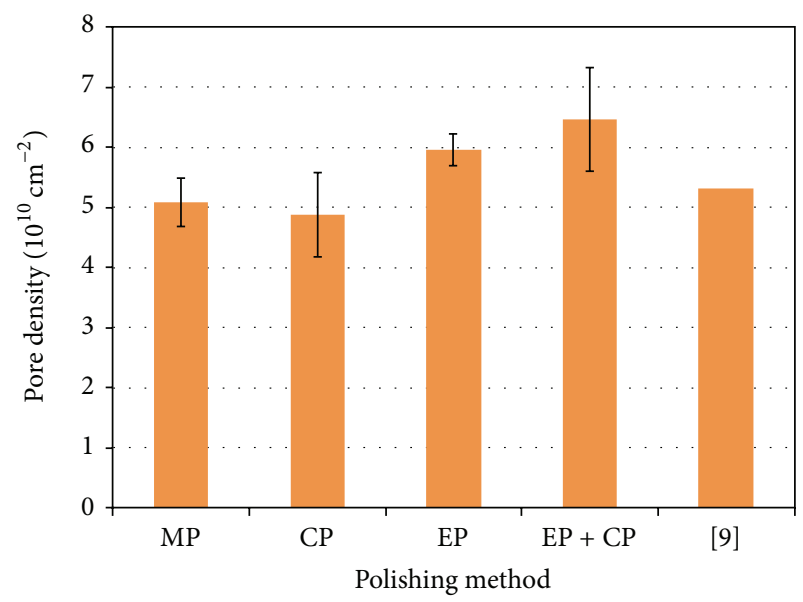

(c)

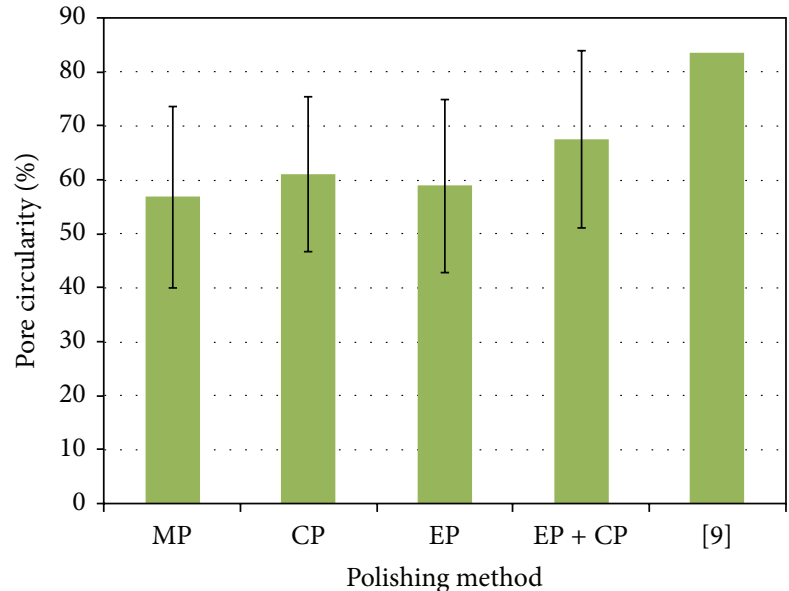

(b)

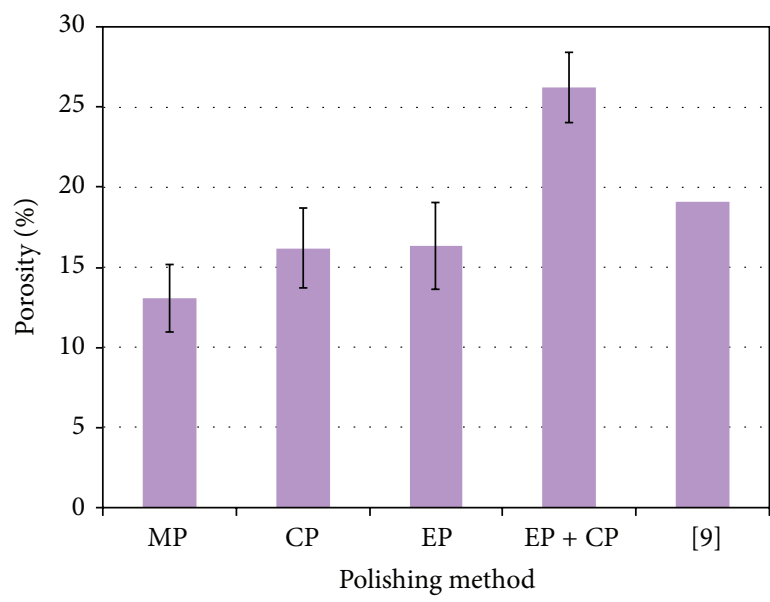

(d)

FIGURE 5: Influence of the polishing method on the pore diameter (a), pore circularity (b), pore density (c), and porosity (d) of anodic titanium dioxide formed by two-step anodization.

array of concaves is present on the titanium surface [9]. These concaves act as nucleation sites for pore growth in the second anodizing step. Therefore, independently of the used polishing method, the similar concave pattern on $\mathrm{Ti}$ is present after the first and before the second anodizing step.

3.3. Polishing Effect on the ATO Structure. To study the influence of used polishing method on the surface characteristics of anodic titanium dioxide, two-step anodizations were performed at $60 \mathrm{~V}$ and $20^{\circ} \mathrm{C}$. An average cell size, pore diameter, pore density, pore circularity, and porosity of the ATO layers were calculated from SEM images similarly as described previously [9]. The cell size of ATO layers was calculated from both top-view and bottom-view SEM images. For a given polishing method, the average cell size in the ATO lattice was calculated from fast Fourier transforms of 6 different top-view or bottom-view SEM images. For each FFT pattern, 3 main profiles along the FFT intensity were constructed. In this way, for each polishing method, the average value of the main distance of the lattice in the inverse space was estimated on the basis of 18 measurements. The results obtained from the top-view and bottom-view calculations are presented in Figure 4. In addition, these results are compared with the data previously reported for top-view measurements [9].

As can be seen, the biggest and the smallest cells are formed on Ti surfaces which were subjected to the MP and $\mathrm{CP}$ procedures, respectively. The same conclusion on MP influence on the cell size was drawn above from the analysis of Figure 2. For other polishing methods, top-view calculations gave similar cell sizes and these results are in agreement with the previously reported data. The excellent agreement between the $\mathrm{EP}+\mathrm{CP}$ and literature values is clearly visible. It is worth noting that the cell sizes calculated from the bottom-view images are slightly smaller than those estimated from top-view images. Figure 4 shows also that the worst and the best uniformity of cell sizes (the highest and the lowest standard deviations) are observed for the CP and EP or EP + $\mathrm{CP}$ pretreatments, respectively. 


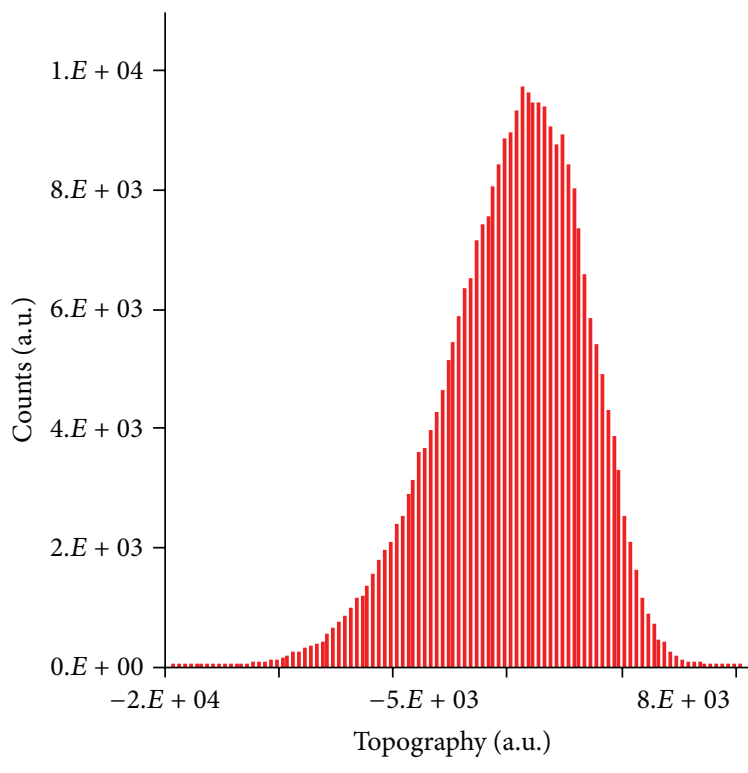

(a)

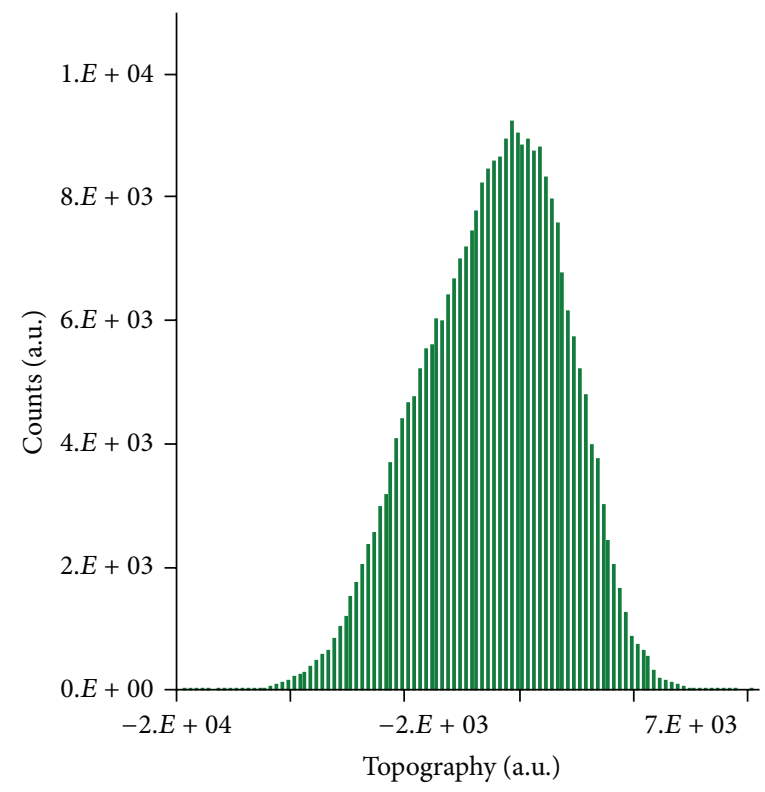

(c)

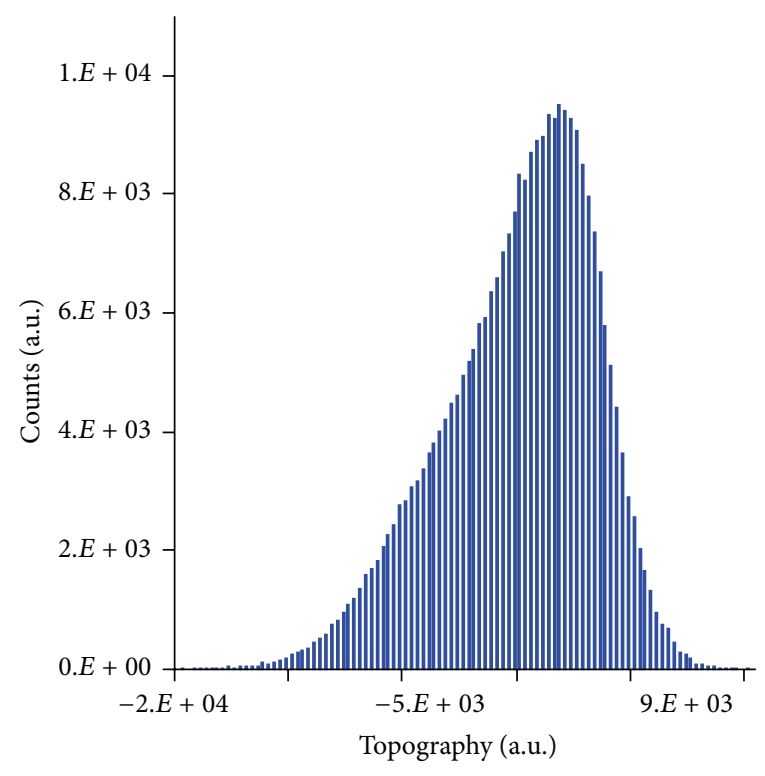

(b)

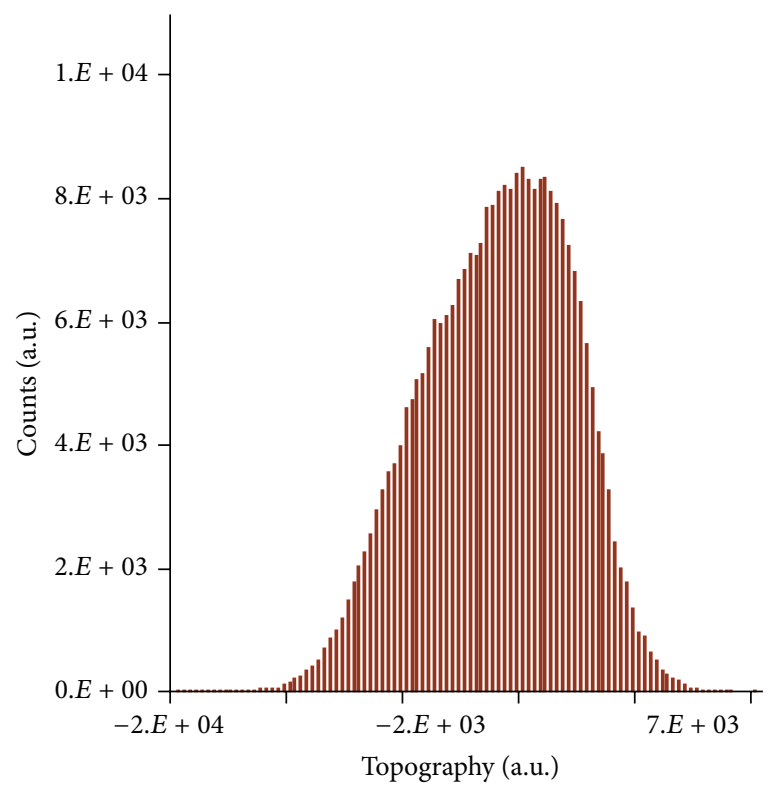

(d)

FIGURE 6: Typical surface-height distribution diagrams derived from the bottom-view SEM images for different polishing procedures: MP (a), CP (b), EP (c), and EP + CP (d).

The average pore diameter, pore circularity, pore density, and porosity of ATO layers were calculated from 6 different SEM top-view images using ImageJ software [47]. The density of pores was calculated as a total number of pores occupying a given surface area of the SEM image. For the pore circularity estimation, it was assumed that the pore circularity of $100 \%$ indicates a perfect circle, while the value close to $0 \%$ indicates an elongated polygon. The results of performed calculations are shown in Figure 5. The data were compared with typical values reported in the literature [9].

The pore diameter values calculated for the MP and EC methods differ slightly from the data reported in the literature. The closest value is achieved for the $\mathrm{EP}+\mathrm{CP}$ method. The highest circularity of pores, pore density, and porosity of the ATO layer are observed for the EP + CP method. However, these results differ from the literature data reported previously. The high porosities of the $\mathrm{EP}+\mathrm{CP}$ and EP samples are a direct consequence of high pore density observed on ATO surfaces. As can be seen in Figure 2(d), the ATO surface pretreated by EP + CP has a complex structure. A mesoporous layer with subpores underneath is present on the ATO surface. This top mesoporous ATO layer is a rutile phase formed during electrochemical oxidation or can be formed as a result of disturbed equilibrium between the rate of chemical etching of titania and rate of precipitation of hydrous titanium dioxide caused by hydrolysis of $\mathrm{Ti}^{4+}[44]$. 


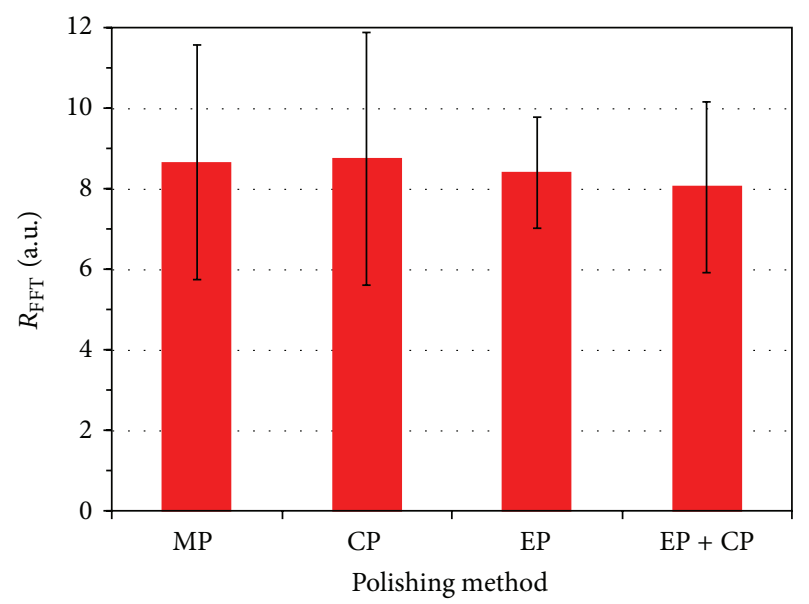

(a)

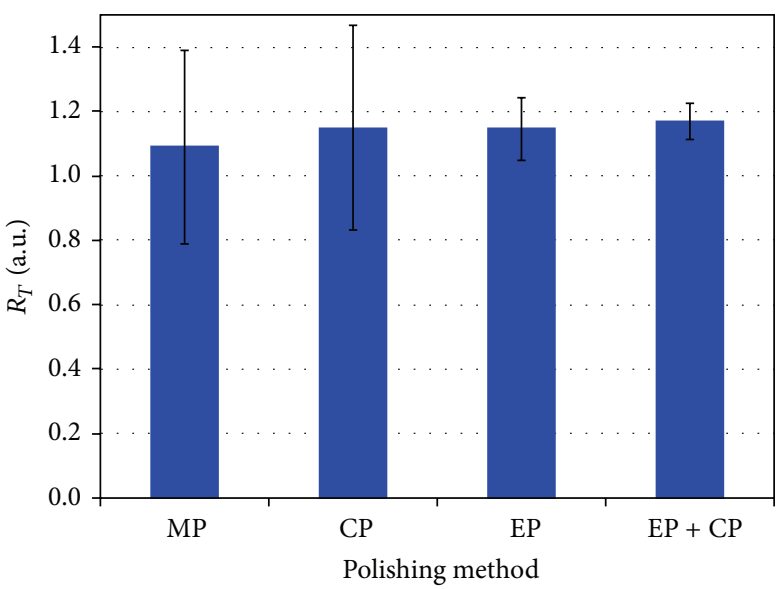

(b)

FIGURE 7: Average regularity ratios derived from FFT images (a) and topography distribution diagrams (b) for different polishing methods.

Nevertheless, this phenomenon is especially important in case of the EP and EP + CP samples.

In summary, the mechanically polished sample is characterized by the largest cell size and the smallest pore diameter, pore circularity, and porosity. The chemically polished and electropolished samples have similar cell sizes, pore diameters, pore circularities, and porosities. For the EP + CP sample, the cell size and pore diameter are almost identical to the literature values, while the porosity and pore density are the highest among all data presented here.

\subsection{Polishing Effect on the Cell Arrangement. A special} emphasis was put on the investigation of the effect of used polishing method on the cell arrangement in ATO layers. Since the morphology of top-view SEM images does not provide useful information about the cell order (see Figures 2(a)-2(d)), the morphology of bottom-view SEM images was deeply analyzed. As was previously discussed (see Figure 2), 2D FFT images can provide some brief and qualitative information about the regularity of cell arrangement, but a quantitative differentiation is not possible. Therefore, using the intensity profiles of FFT images, a detailed analysis of cell arrangement was performed as described previously [9]. A regularity ratio $\left(R_{\mathrm{FFT}}\right)$, defined as a ratio of the maximum intensity of the FFT peak to its width at half-maximum, was calculated from the average FFT profile for various polishing methods. For all polishing methods, the regularity ratio was derived from the SEM images acquired at the same magnification.

On the other hand, the average regularity ratio $\left(R_{T}\right)$ can be estimated in the same manner from the surface-height distribution diagrams (Figure 6) calculated from bottomview SEM images using appropriate software [47].

For typical diagrams (Figure 6), the surface heights are almost equally distributed among positive and negative regions along the topography axis. However, some significant differences in peak intensities and height distributions exist (compare, e.g., Figures 6(a) and 6(d)). The surface-height distribution diagrams were generated for each polishing method and for each SEM image taken at different magnifications. Therefore, for a given polishing method, the average $R_{T}$ value is based on 18 independent measurements.

Figure 7 shows the regularity ratios calculated using both methods based on FFT images $\left(R_{\mathrm{FFT}}\right)$ and topography distributions $\left(R_{T}\right)$. It was previously mentioned that the morphology of bottom-side of oxide layer is not significantly affected by the polishing method (Figures $2(\mathrm{e})-2(\mathrm{~h})$ ). This conclusion is reaffirmed by the data collected in Figure 7. It can be seen that the average values of both regularity ratios, $R_{\mathrm{FFT}}$ and $R_{T}$, do not change considerably when considering different polishing methods.

\section{Conclusion}

In this study, different polishing methods, mechanical, chemical, electrochemical, and a combination of electrochemical with chemical methods, were investigated to establish whether they represent efficient pretreating procedures of $\mathrm{Ti}$ preparation before the formation of anodic titanium dioxide layers. It was established that current density-time curves can provide useful information about retardation of pore nucleation on the anodized surface. While the top morphology of ATO layer and, consequently, the structural features such as cell size, pore diameter, pore circularity, pore density, and porosity are dependent upon the used polishing method, the order in cell arrangement is similar for all polishing methods as demonstrated from the bottom-side morphology of ATO layer formed after the second anodization. Finally, all four studied polishing methods were equally effective when using two-step anodization procedure for ATO formation.

\section{Highlights}

Consider the following

(i) Different methods of the Ti foil polishing were examined. 
(ii) Effect of polishing method on anodic titania formation was studied.

(iii) The surface structure of $\mathrm{TiO}_{2}$ is affected by polishing pretreatment.

(iv) The regularity of cell arrangement is independent of used polishing method.

\section{Conflict of Interests}

The authors declare that there is no conflict of interests regarding the publication of this paper.

\section{Acknowledgments}

Magdalena Jarosz acknowledges the financial support from the project Interdisciplinary PhD Studies "Molecular sciences for medicine" (cofinanced by the European Social Fund within the Human Capital Operational Programme). This research was partially supported by the National Science Centre, Poland (Grant no. N N204 213340).

\section{References}

[1] J. M. Macak, M. Zlamal, J. Krysa, and P. Schmuki, "Self-organized $\mathrm{TiO}_{2}$ nanotube layers as highly efficient photocatalysts," Small, vol. 3, no. 2, pp. 300-304, 2007.

[2] S. P. Albu, A. Ghicov, J. M. Macak, R. Hahn, and P. Schmuki, "Self-organized, free-standing $\mathrm{TiO}_{2}$ nanotube membrane for flow-through photocatalytic applications," Nano Letters, vol. 7, no. 5, pp. 1286-1289, 2007.

[3] S.-S. Kim, S.-I. Na, and Y.-C. Nah, " $\mathrm{TiO}_{2}$ nanotubes decorated with $\mathrm{ZnO}$ rod-like nanostructures for efficient dye-sensitized solar cells," Electrochimica Acta, vol. 58, pp. 503-509, 2011.

[4] C. Rho, J.-H. Min, and J. S. Suh, "Barrier layer effect on the electron transport of the dye-sensitized solar cells based on $\mathrm{TiO}_{2}$ nanotube arrays," Journal of Physical Chemistry C, vol. 116, no. 12, pp. 7213-7218, 2012.

[5] P. Roy, C. Das, K. Lee et al., "Oxide nanotubes on Ti-Ru alloys: strongly enhanced and stable photoelectrochemical activity for water splitting," Journal of the American Chemical Society, vol. 133, no. 15, pp. 5629-5631, 2011.

[6] E. Balaur, J. M. MacAk, L. Taveira, and P. Schmuki, “Tailoring the wettability of $\mathrm{TiO}_{2}$ nanotube layers," Electrochemistry Communications, vol. 7, no. 10, pp. 1066-1070, 2005.

[7] Q. Zheng, B. Zhou, J. Bai et al., "Self-organized $\mathrm{TiO}_{2}$ nanotube array sensor for the determination of chemical oxygen demand," Advanced Materials, vol. 20, no. 5, pp. 1044-1049, 2008.

[8] G. K. Mor, M. A. Carvalho, O. K. Varghese, M. V. Pishko, and C. A. Grimes, "A room-temperature $\mathrm{TiO}_{2}$-nanotube hydrogen sensor able to self-clean photoactively from environmental contamination," Journal of Materials Research, vol. 19, no. 2, pp. 628-634, 2004.

[9] G. K. Mor, K. Shankar, M. Paulose, O. K. Varghese, and C. A. Grimes, "Enhanced photocleavage of water using titania nanotube arrays," Nano Letters, vol. 5, no. 1, pp. 191-195, 2005.

[10] U. Diebold, “The surface science of titanium dioxide," Surface Science Reports, vol. 48, no. 5-8, pp. 53-229, 2003.
[11] V. Zwilling, E. Darque-Ceretti, A. Boutry-Forveille, D. David, M. Y. Perrin, and M. Aucouturier, "Structure and physicochemistry of anodic oxide films on titanium and TA6V alloy," Surface and Interface Analysis, vol. 27, no. 7, pp. 629-637, 1999.

[12] G. D. Sulka, J. Kapusta-Kołodziej, A. Brzózka, and M. Jaskuła, "Fabrication of nanoporous $\mathrm{TiO}_{2}$ by electrochemical anodization," Electrochimica Acta, vol. 55, no. 14, pp. 4359-4367, 2010.

[13] J. H. Jung, H. Kobayashi, K. J. C. van Bommel, S. Shinkai, and T. Shimizu, "Creation of novel helical ribbon and doublelayered nanotube $\mathrm{TiO}_{2}$ structures using an organogel template," Chemistry of Materials, vol. 14, no. 4, pp. 1445-1447, 2002.

[14] Y.-H. Chang, C.-M. Liu, C. Chen, and H.-E. Cheng, "The heterojunction effects of $\mathrm{TiO}_{2}$ nanotubes fabricated by atomic layer deposition on photocarrier transportation direction," Nanoscale Research Letters, vol. 7, article 231, 2012.

[15] T. Kasuga, M. Hiramatsu, A. Hoson, T. Sekino, and K. Niihara, "Formation of titanium oxide nanotube," Langmuir, vol. 14, no. 12, pp. 3160-3163, 1998.

[16] Z. R. Tian, J. A. Voigt, J. Liu, B. McKenzie, and H. F. Xu, "Large oriented arrays and continuous films of $\mathrm{TiO}_{2}$-based nanotubes," Journal of the American Chemical Society, vol. 125, no. 41, pp. 12384-12385, 2003.

[17] H. Masuda and K. Fukuda, "Ordered metal nanohole arrays made by a two-step replication of honeycomb structures of anodic alumina," Science, vol. 268, no. 5216, pp. 1466-1468, 1995.

[18] J. Oh, Y. Tak, and J. Lee, "Electrodeposition of $\mathrm{Cu}_{2} \mathrm{O}$ nanowires using nanoporous alumina template," Electrochemical and SolidState Letters, vol. 7, pp. C21-C30, 2004.

[19] K. Nielsch, F. Müller, A.-P. Li, and U. Gösele, "Uniform nickel deposition into ordered alumina pores by pulsed electrodeposition," Advanced Materials, vol. 12, no. 8, pp. 582-586, 2000.

[20] M. R. Hoffmann, S. T. Martin, W. Choi, and D. W. Bahnemann, "Environmental applications of semiconductor photocatalysis," Chemical Reviews, vol. 95, no. 1, pp. 69-96, 1995.

[21] J. M. Macak, H. Hildebrand, U. Marten-Jahns, and P. Schmuki, "Mechanistic aspects and growth of large diameter selforganized $\mathrm{TiO}_{2}$ nanotubes," Journal of Electroanalytical Chemistry, vol. 621, no. 2, pp. 254-266, 2008.

[22] Q. Cai, M. Paulose, O. K. Varghese, and C. A. Grimes, "The effect of electrolyte composition on the fabrication of self-organized titanium oxide nanotube arrays by anodic oxidation," Journal of Materials Research, vol. 20, no. 1, pp. 230-236, 2005.

[23] S. Berger, J. Kunze, P. Schmuki et al., "Influence of water content on the growth of anodic $\mathrm{TiO}_{2}$ nanotubes in fluoride-containing ethylene glycol electrolytes," Journal of the Electrochemical Society, vol. 157, no. 1, pp. C18-C23, 2010.

[24] L. V. Taveira, J. M. Macák, H. Tsuchiya, L. F. P. Dick, and P. Schmuki, "Initiation and growth of self-organized $\mathrm{TiO}_{2}$ nanotubes anodically formed in $\mathrm{NH}_{4} \mathrm{~F} /\left(\mathrm{NH}_{4}\right)_{2} \mathrm{SO}_{4}$ electrolytes," Journal of the Electrochemical Society, vol. 152, no. 10, pp. B405B410, 2005.

[25] S. Li, G. Zhang, D. Guo, L. Yu, and W. Zhang, "Anodization fabrication of highly ordered $\mathrm{TiO}_{2}$ nanotubes," Journal of Physical Chemistry C, vol. 113, no. 29, pp. 12759-12765, 2009.

[26] S. Yeonmi and L. Seonghoon, "Self-organized regular arrays of anodic $\mathrm{TiO}_{2}$ nanotubes," Nano Letters, vol. 8, no. 10, pp. 31713173, 2008.

[27] B. Chen, K. Lu, and Z. Tian, "Gradient and alternating diameter nanopore templates by focused ion beam guided anodization," Electrochimica Acta, vol. 56, no. 1, pp. 435-440, 2010. 
[28] B. Chen, K. Lu, and Z. Tian, "Novel patterns by focused ion beam guided anodization," Langmuir, vol. 27, no. 2, pp. $800-$ 808, 2011.

[29] D. Ma, S. Li, and C. Liang, "Electropolishing of high-purity aluminium in perchloric acid and ethanol solutions," Corrosion Science, vol. 51, no. 4, pp. 713-718, 2009.

[30] O. Jessensky, F. Müller, and U. Gösele, "Self-organized formation of hexagonal pore structures in anodic alumina," Journal of the Electrochemical Society, vol. 145, no. 11, pp. 3735-3740, 1998.

[31] Z.-P. Tian, K. Lu, and B. Chen, "Unique nanopore pattern formation by focused ion beam guided anodization," Nanotechnology, vol. 21, no. 40, Article ID 405301, 2010.

[32] Z. Tian, K. Lu, and B. Chen, "Fundamental mechanisms of focused ion beam guided anodization," Journal of Applied Physics, vol. 108, no. 9, Article ID 094306, 2010.

[33] O. Piotrowski, C. Madore, and D. Landolt, "The mechanism of electropolishing of titanium in methanol-sulfuric acid electrolytes," Journal of the Electrochemical Society, vol. 145, no. 7, pp. 2362-2369, 1998.

[34] L. Neelakantan and A. W. Hassel, "Rotating disc electrode study of the electropolishing mechanism of NiTi in methanolic sulfuric acid," Electrochimica Acta, vol. 53, no. 2, pp. 915-919, 2007.

[35] K. Fushimi, M. Stratmann, and A. W. Hassel, "Electropolishing of NiTi shape memory alloys in methanolic $\mathrm{H}_{2} \mathrm{SO}_{4}$," Electrochimica Acta, vol. 52, no. 3, pp. 1290-1295, 2006.

[36] C.-C. Chen, J.-H. Chen, C.-G. Chao, and W. C. Say, "Electrochemical characteristics of surface of titanium formed by electrolytic polishing and anodizing," Journal of Materials Science, vol. 40, no. 15, pp. 4053-4059, 2005.

[37] E. Mahé and D. Devilliers, "Surface modification of titanium substrates for the preparation of noble metal coated anodes," Electrochimica Acta, vol. 46, no. 5, pp. 629-636, 2001.

[38] J. B. Mathieu, H. J. Mathieu, and D. Landolt, "Electropolishing of titanium in perchloric acid-acetic acid solution. I. Auger electron spectroscopy study of anodic films," Journal of the Electrochemical Society, vol. 125, no. 7, pp. 1039-1043, 1978.

[39] J. B. Mathieu and D. Landolt, "Electropolishing of titanium in perchloric acid-acetic acid solution II . Polarization behavior and stoichiometry," Journal of the Electrochemical Society, vol. 125, no. 7, pp. 1044-1049, 1978.

[40] K. Tajima, M. Hironaka, K.-K. Chen, Y. Nagamatsu, H. Kakigawa, and Y. Kozono, "Electropolishing of CP titanium and its alloys in an alcoholic solution-based electrolyte," Dental Materials Journal, vol. 27, no. 2, pp. 258-265, 2008.

[41] M. Yu, J. Yi, J. Liu, S. Li, G. Wu, and L. Wu, "Effect of electropolishing on electrochemical behaviours of titanium alloy Ti-10V2Fe-3Al," Journal of Wuhan University of Technology-Mater. Sci. Ed., vol. 26, no. 3, pp. 469-477, 2011.

[42] B. G. Lee, S.-Y. Hong, J. E. Yoo, and J. Choi, "Electropolishing for the formation of anodic nanotubular $\mathrm{TiO}_{2}$ with uniform length and density," Applied Surface Science, vol. 257, no. 16, pp. 71907194, 2011.

[43] G. R. Dale, J. W. J. Hamilton, P. S. M. Dunlop, P. Lemoine, and J. A. Byrne, "Electrochemical growth of titanium oxide nanotubes: the effect of surface roughness and applied potential," Journal of Nanoscience and Nanotechnology, vol. 9, no. 7, pp. 4215-4219, 2009.

[44] D. Kim, A. Ghicov, and P. Schmuki, “ $\mathrm{TiO}_{2}$ Nanotube arrays: elimination of disordered top layers ('nanograss') for improved photoconversion efficiency in dye-sensitized solar cells," Electrochemistry Communications, vol. 10, no. 12, pp. 1835-1838, 2008.

[45] K. Lu, Z. Tian, and J. A. Geldmeier, "Polishing effect on anodic titania nanotube formation," Electrochimica Acta, vol. 56, no. 17, pp. 6014-6020, 2011.

[46] I. Horcas, R. Fernández, J. M. Gómez-Rodríguez, J. Colchero, J. Gómez-Herrero, and A. M. Baro, "WSXM: a software for scanning probe microscopy and a tool for nanotechnology," Review of Scientific Instruments, vol. 78, no. 1, Article ID 013705, 2007.

[47] Image J, National Institute of Mental Health, Bethesda, Md, USA, http://rsb.info.nih.gov/ij/.

[48] P. Roy, S. Berger, and P. Schmuki, " $\mathrm{TiO}_{2}$ nanotubes: synthesis and applications," Angewandte Chemie International Edition, vol. 50, no. 13, pp. 2904-2939, 2011. 

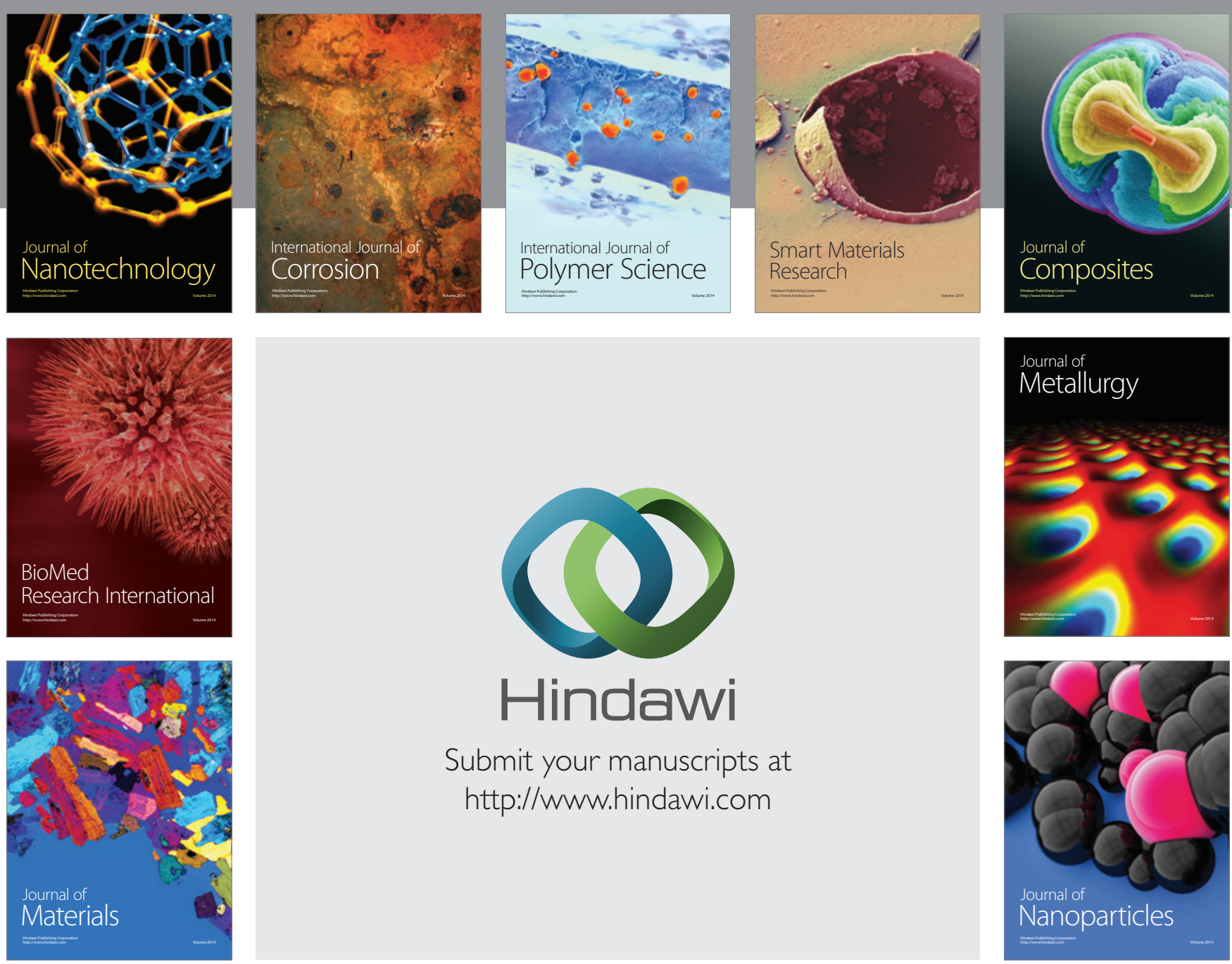

Submit your manuscripts at http://www.hindawi.com
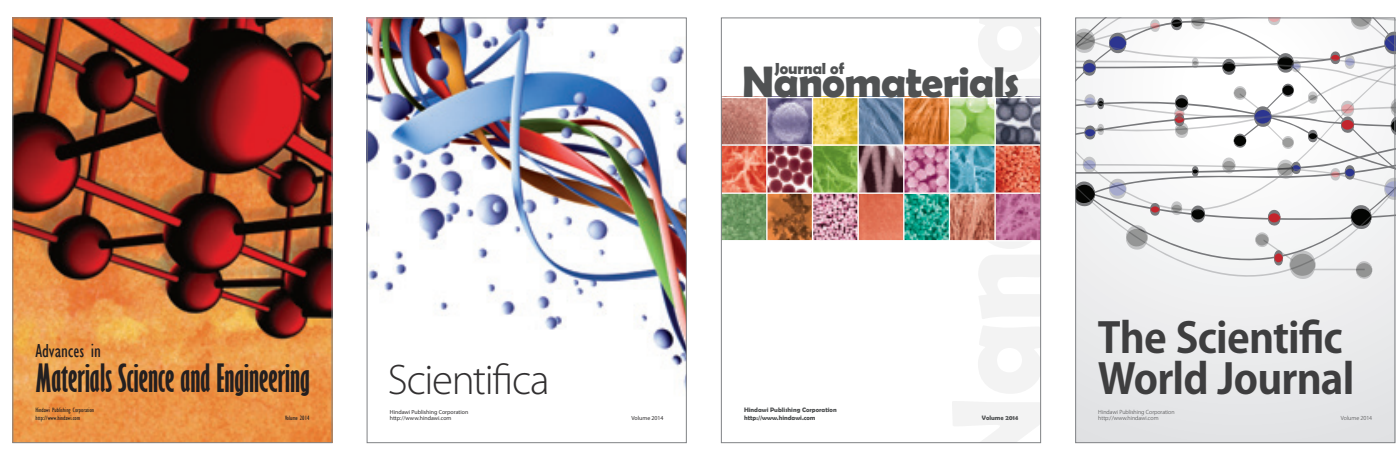

\section{The Scientific World Journal}
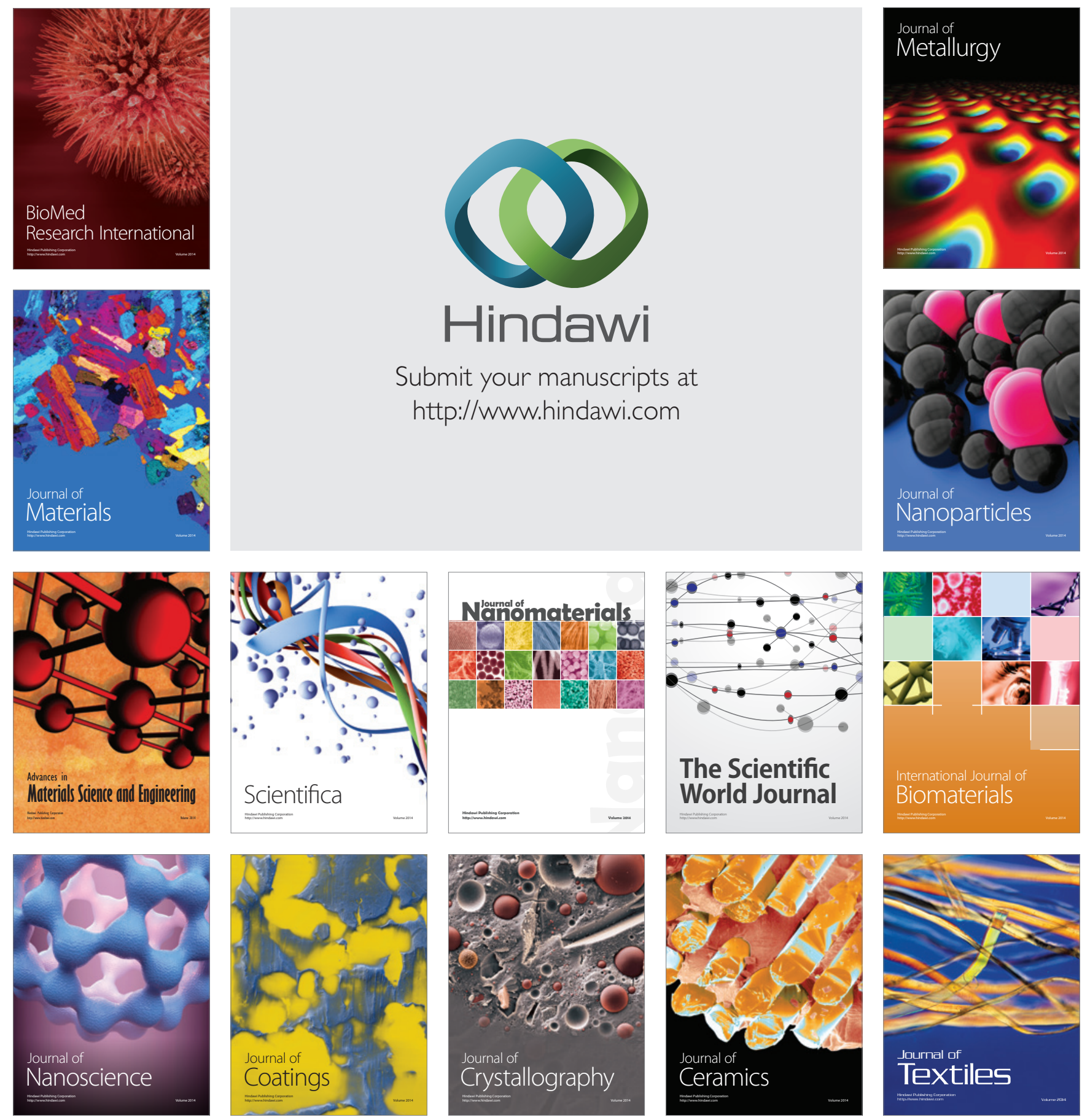